\title{
Coulomb and even-odd effects in cold and super-asymmetric fragmentation for thermal neutron induced fission of ${ }^{235} \mathrm{U}$
}

\author{
M. Montoya \\ Instituto Peruano de Energía Nuclear, Canadá 1470, San Borja, Lima, Peru \\ Universidad Nacional de Ingeniería, Av. Túpac Amaru 210, Rímac, Lima, Peru
}

DOI: https://doi.org/10.33017/RevECIPeru2015.0004/

\begin{abstract}
The Coulomb effects hypothesis is used to interpret even-odd effects of maximum total kinetic energy as a function of mass and charge of fragments from thermal neutron induced fission of ${ }^{235} \mathrm{U}$. Assuming spherical fragments at scission, the Coulomb interaction energy between fragments $\left(C_{\mathrm{sph}}\right)$ is higher than the $Q$-value, the available energy. Therefore at scission the fragments must be deformed, so that the Coulomb interaction energy does not exceed the $Q$-value. The fact that the even-odd effects in the maximum total kinetic energy as a function of the charge and mass, respectively, are lower than the even-odd effects of $Q$ is consistent with the assumption that odd mass fragments are softer than the even-even fragments.

Even-odd effects of charge distribution in super asymmetric fragmentation also are interpreted with the Coulomb effect hypothesis. Because the difference between $C_{\mathrm{sph}}$ and $Q$ increases with asymmetry, fragmentations require higher total deformation energy to occur. Higher deformation energy of the fragments implies lower free energy to break pairs of nucleons. This explains why in the asymmetric fragmentation region, the even-odd effects of the distribution of proton number and neutron number increases with asymmetry. Based on a similar reasoning, a prediction of a relatively high even-odd effect in symmetric fragmentations is proposed.
\end{abstract}

Keywords: cold fission, asymmetric fragmentation, symmetric fission, kinetic energy, uranium 235

\section{Resumen}

La hipótesis del efecto Coulomb es usado para interpretar los efectos par-impar sobre la energía total máxima en función de la masa y carga de los fragmentos de la fisión inducida por neutrones térmicos del ${ }^{235} U$. Suponiendo que en la escisión los fragmentos son esféricos, la energía de interacción coulombiana entre fragmentos $\left(C_{\mathrm{sph}}\right)$ es mayor que el valor $Q$, la energía disponible. Entonces, los fragmentos en la escisión deben estar deformados, de modo que la energía de interacción coulombiana no exceda el valor $Q$ de la reacción. El hecho de que los efectos par-impar sobre el valor máximo de la energía cinética total en función de la carga y la masa, respectivamente, son menores que los efectos par-impar de $Q$ es consistente con la suposición que los fragmentos con masa impar son más blandos que los fragmentos par-par.

Los efectos par-impar de la distribución de carga en las fragmentaciones super asimétricas también son interpretados con la hipótesis del efecto Coulomb. Debido a que la diferencia entre $C_{\mathrm{sph}}$ y $Q$ crece con la asimetría, las fragmentaciones requieren mayor energía total de deformación para ocurrir. Mayor energía de deformación de los fragmentos implica menor energía libre para romper pares de nucleones. Esto explica por qué en la region de asimetría, los efectos par-impar en la distribución de número de protons y número de neutrones crece con la asimetría. Basado en un razonamiento similar, se predice un alto efecto par-impar en las fragmentaciones simétricas.

Descriptores: fisión fría, fragmentación asimétrica, fisión simétrica, energía cinética, uranio 235. 


\section{Introduction}

The even-odd effects in the distribution of kinetic energy, charge and mass are among the properties that have generated controversy in the study of fragments from thermal neutron induced fission of actinides. To describe these effects is useful to recall some definitions related to them. Let be a fissile nucleus with charge $Z_{\mathrm{f}}$ and mass $A_{\mathrm{f}}$ that splits in a light fragment with $Z_{\mathrm{L}}$ protons, $N_{\mathrm{L}}$ neutrons (number of nucleons $A_{\mathrm{L}}=Z_{\mathrm{L}}+N_{\mathrm{L}}$ ) and a heavy fragment with $Z_{\mathrm{H}}$ protons, $N_{\mathrm{H}}$ neutrons (number of nucleons $A_{\mathrm{H}}=Z_{\mathrm{H}}$ $+N_{\mathrm{H}}$ ). These numbers obey the following relations:

$$
Z_{\mathrm{f}}=Z_{\mathrm{L}}+Z_{\mathrm{H}}
$$

and

$$
A_{\mathrm{f}}=A_{\mathrm{L}}+A_{\mathrm{H}} .
$$

Based on these relations, to identify the two complementary fragments from a fission event it is enough to know the charge $(Z)$ and the proton number $(N)$ or the mass number $(A)$ of the light fragment.

After scission, the light and heavy fragments acquire kinetic energies $K_{\mathrm{L}}, K_{\mathrm{H}}$ and excitation energies $X_{\mathrm{L}}$, $X_{\mathrm{H}}$, respectively. Thus, the total kinetic energy $(K)$ and the total excitation energy $(X)$ are

and

$$
K=K_{\mathrm{L}}+K_{\mathrm{H}}
$$

$$
X=X_{\mathrm{L}}+X_{\mathrm{H}} \text {, }
$$

respectively. These quantities are limited by the energy balance equation:

$$
Q=K+X,
$$

where $Q$ is the available energy of the reaction.

At the scission point, the available energy is spent into deformation energy $(D)$, Coulomb interaction energy $(C)$ and free energy $(F)$ :

$$
Q=C+D+F \text {. }
$$

The free energy is partitioned into intrinsic energy $\left(X^{*}\right)$ and total pre-scission energy of fragments $\left(K_{S}\right)$ :

$$
F=X^{*}+K_{s} .
$$

The preference for even proton numbers in the fission fragments has been well established [1], leading to the definition of the even-odd effect of charge distribution $(\delta Z)$ :

$$
\delta Z=\frac{Y_{e}^{Z}-Y_{o}^{Z}}{Y_{e}^{Z}+Y_{o}^{Z}}
$$

where $Y_{e}^{Z}$ y $Y_{o}^{Z}$ are the yield of fragments with even and odd proton numbers, respectively. Similarly are defined the even-odd effect in the distribution neutron number $(\delta N)$ and nucleon numbers $(\delta A)$, respectively.

For a given fragmentation corresponding to proton and mass numbers $Z$ and $A$, respectively, the maximum total kinetic energy $\left(K_{\max }\right)$ is reached by a configuration that at scission acquires a maximum Coulomb interaction energy $\left(C_{\max }\right)$ and a minimum total deformation energy $\left(D_{\min }\right)$, limited by the equation

$$
Q=C_{\max }+D_{\min }
$$

Because Coulomb repulsion between fragments, Coulomb interaction energy becomes total kinetic energy, so that:

$$
K_{\text {max }}=C_{\text {max }}=Q-D_{\text {min }} .
$$

Let be $A$ an odd nucleon number of the light fragment, the local even-odd effect in the maximum $Q$-value $\left(Q_{\max }^{A}\right)$ as a function of mass, is defined as

$$
\delta_{A} Q_{\max }=\frac{Q_{\max }^{A-1}+Q_{\max }^{A+1}}{2}-Q_{\max }^{A} .
$$

In average $\delta_{A} Q_{\max }$ is positive. Similarly there are local even-odd effects in $Q_{\max }$ as a function of proton number $\left(\delta_{Z} Q_{\max }\right)$ and neutron number $\left(\delta_{N} Q_{\max }\right)$, respectively.

Because the even-odd effect of charge and mass distribution, respectively, increases with the fragment kinetic energy [1], a positive local even-odd effect in the maximum total kinetic energy as a function of mass,

$$
\delta_{A} K_{\max }=\frac{K_{\max }^{A-1}+K_{\max }^{A+1}}{2}-K_{\max }^{A},
$$

is expected. Similarly is expected positive values of $\delta_{Z} K_{\max }$ and $\delta_{N} K_{\max }$, which correspond to even-odd effects in the maximum total kinetic energy as a function of proton and neutron numbers, respectively.

Surprisingly, when C. Signarbieux et al. found the evidence of the existence of cold fission, in which the excitation energy is not enough for the fragments to emit neutrons, they do not find a significant even-odd 
effect in the distribution of the nucleon numbers $(\delta A \cong 0)$. This set a controversy in those authors that, based on the even-odd effects in proton and neutron number distribution, respectively, supported the hypothesis that the fission process is superfluid [2]. However, in 1981, M. Montoya deduced [3, 4] that

$$
\delta A=\delta Z+\delta N-1,
$$

which was confirmed by $\mathrm{H}$. Nifenecker [5]. That relation shows that there is no contradiction between a null even-odd effect in $A$ distribution and no-null even-odd effects in $Z$ and $N$ distributions, respectively.

In 1991, based on data communicated by $C$. Signarbieux et al., F. Gönnenwein and B. Börsig show that the minimum excitation energy,

$$
X_{\min }=Q_{K_{\max }}-K_{\max }
$$

where $\left(Q_{K_{\max }}\right)$ is the $Q$-value corresponding to the charge that maximizes the total kinetic energy, is lower for the odd than for the even proton numbers [6]. This result encourages research on even-odd effects, which leads to review the existent results and interpretations about even-odd effects in the distribution of mass, charge and maximum total kinetic energy of fragments.

\section{Even-odd effects in the maximum total kinetic energy}

Taking into account the $3 \times 10^{6}$ events from thermal neutron induced fission of ${ }^{235} \mathrm{U}$, obtained in 1981 by Signarbieux et al. [2], in $1984 \mathrm{M}$. Montoya presents the curve of threshold values for the 10 events with the highest total kinetic energy values [7]. These threshold values are assumed to correspond to the maximum total kinetic energy for each isobaric fragmentation. See Fig. 1.

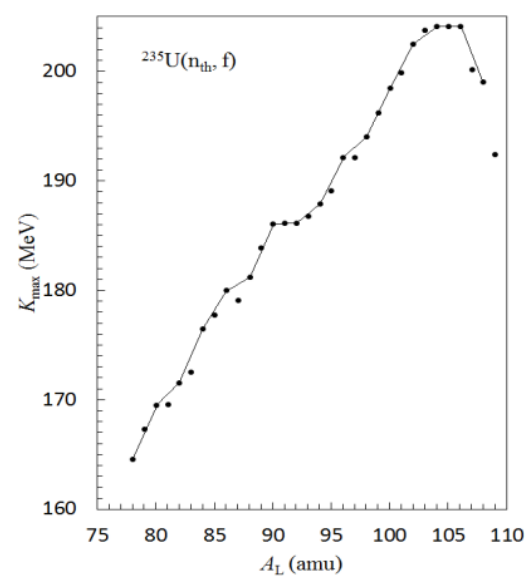

Fig. 1: Thermal neutron induced fission of ${ }^{235} \mathrm{U}$. Threshold values for which there are only 10 events with higher values of total kinetic energy of fragments. The total number of events is $3 \times 10^{6}$. Data taken from Ref. [7]. The average of even-odd effect on $K_{\max }$ as a function of the fragments mass is $0.5 \mathrm{MeV}$.

If one draws an straight line segments between the points corresponding to even nucleon numbers $\left(A_{\mathrm{L}}\right)$, the points corresponding to odd nucleon numbers are generally below that line, which is an indication that the average of $\delta_{A} K_{\max }$ is positive. Actually, the average value of $\delta_{A} K_{\max }$ is $0.5 \mathrm{MeV}$.

In Fig. 2 one presents the maximum total kinetic energy as a function of charge and mass of fragments, obtained in 1986 by G. Simon et al. [8]. The cases where the charge that maximizes the total kinetic energy is odd are indicated. The average even-odd effect in the maximum total kinetic energy as a function of mass $\left(\delta_{A} K_{\max }\right)$ is $1 \mathrm{MeV}$. If we take groups of 3 masses corresponding to three different neighboring charges, the average of $\delta_{Z} K_{\max }$ is $=0.66$ $\mathrm{MeV}$. Note that in these results for the masses 85 and 86 , the charge that maximizes the total kinetic energy in both cases is 35 .

In Fig. 3 the curve associated to the maximum total kinetic energy as a function of fragment mass communicated by C. Signarbieux is presented by $\mathrm{F}$. Gönnenwein and B. Börsig [6]. In Fig. 4 one can see the charges maximizing the total kinetic energy and the charges that maximize the available energy, respectively. The $Q$-values are calculated using the mass table from Ref. [9].

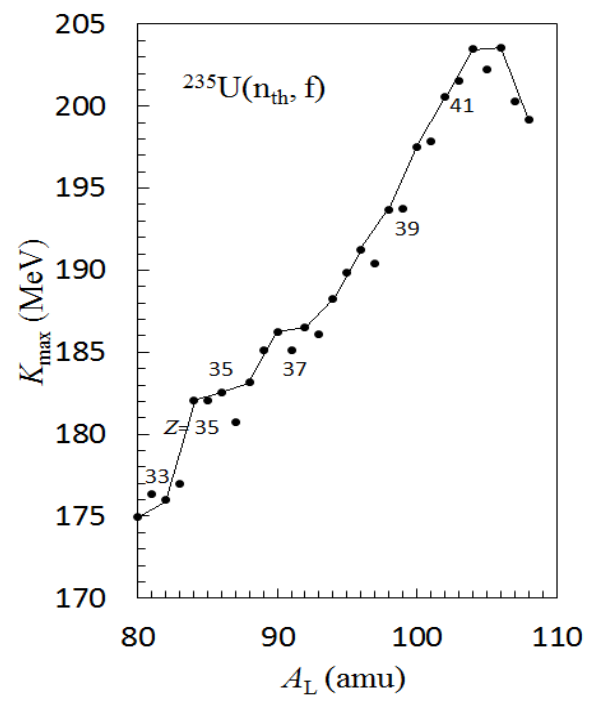

Fig. 2: Thermal neutron induced fission of ${ }^{235} U$. Curve of the maximum total kinetic energy $\left(K_{\max }\right)$ as a function of the light fragment mass number is presented. Taken from Ref. [8]. The charges that 
Revista ECIPerú maximize $K_{\max }$ indicated.

The average of even-odd effect in the maximum total kinetic energy as a function of the mass $\left(\delta_{A} K_{\max }\right)$ is $0.5 \mathrm{MeV}$. If one takes 3 neighboring masses, corresponding to three different charges, in average it results $\delta_{Z} K_{\max }=0.8 \mathrm{MeV}$. Note that the average of odd-even effect on the maximum available energy as a function of mass is $1.6 \mathrm{MeV}$.

To interpret this result one must remember that $Q_{K_{\max }}=C_{\max }+D_{\min }$, regarding which it follows that

$$
\delta_{A} C_{\max }=\delta_{A} K_{\max }=\delta_{A} Q_{K_{\max }}-\delta_{A} D_{\min } .
$$

The experimental result,

$$
\delta_{A} K_{\max }<\delta_{A} Q_{K_{\max }}
$$

implies that

$$
\delta_{A} D_{\min }>0 \text {, }
$$

which suggests that the even-even fragments are harder than odd $A$ fragments, they need higher deformation energy to get the most compact configuration that obeys the relation $C_{\max }=Q_{K_{\max }}-$ $D_{\text {min }}$.

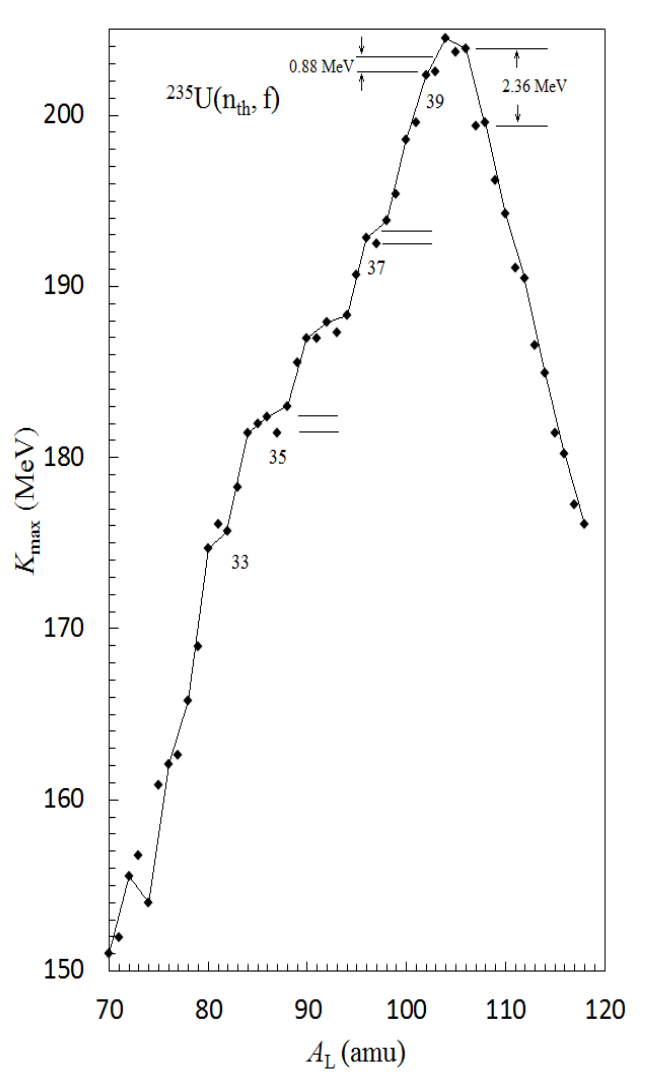

Julio 2015

Fig. 3: Thermal neutron induced fission of ${ }^{235} \mathrm{U}$. Curve of the maximum total kinetic energy $\left(K_{\max }\right)$ as a function of the light fragment mass number is presented. Taken from Ref. [5]. The charges that maximize $K_{\max }$ for each mass fragmentation are indicated.

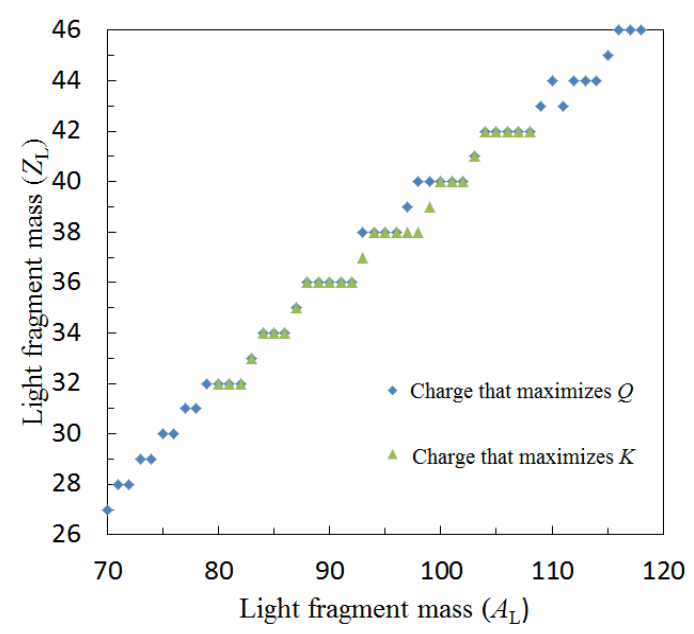

Fig. 4: Thermal neutron induced fission of ${ }^{235} U$. For each light fragment mass the charge that maximizes the $Q$-values (diamonds) and the charge maximizing the $K_{\max }$ values (triangles) are presented. The $Q$ values are calculated using the mass table from Ref. [9]

In 2013, F. Gönnenwein and B. Börsig show that, for mass fragmentations $104 / 132$, the kinetic energy associated to the charge fragmentation $41 / 51$ reach the $Q$-value of the reaction, while the corresponding to the fragmentation $42 / 50$ reaches a total maximum kinetic energy below $3 \mathrm{MeV}$ the corresponding $Q$ value [10]. However, we should note that charge fragmentation $41 / 51$ is more asymmetric than the $42 / 50$ fragmentation. Therefore that result is also consistent with the Coulomb effect: for neighboring masses with similar values of energy available, the more asymmetric fragmentation reaches the higher values of total kinetic energy $[11,12]$.

In general, the results presented by $F$. Gönnenwein and $B$. Börsig show that the charges that maximize the total kinetic energy are the same as the charges that maximize the available energy. Exceptions occur for the masses 93, 96, 97 and 98, whose charges that maximize the available energy are 38, 39, 40 and 40 , whereas the corresponding charges that maximize the total kinetic energy are $36(<38), 38(<39), 38$ and $39(<40)$, respectively. These results are also consistent with the hypothesis of the Coulomb effect $[11,12]$.

3. Coulomb and even-odd effects in superasymmetric fragmentations 
In 1989, J. L. Sida et al. note that in the region of super-asymmetric charge fragmentations, even-odd effect in charge and neutron number yields increase with charge asymmetry [13]. See Fig. 5. This result is consistent with the hypothesis of Coulomb effect. Indeed, if one assumes a scission configuration with spherical fragments whose surfaces, the Coulomb interaction energy is higher than the corresponding $Q$ value. Therefore at scission point the fragments must be deformed so that the energy of Coulomb interaction is lower than the available energy $[11,12]$. For masses lower than 104 the Coulomb interaction energy curve separates from the value $Q$ to the extent that the fragmentation is asymmetric. This implies that the higher the asymmetry, the higher must be the deformation energy of the fission fragments will be to make the fission possible. Therefore, the process has lower free energy,

$$
F=Q-C-D \text {. }
$$

A lower free energy implies a lower probability to break pairs of nucleons, therefore a higher even-odd effect in yields of charge. This is precisely what experimentally is observed.

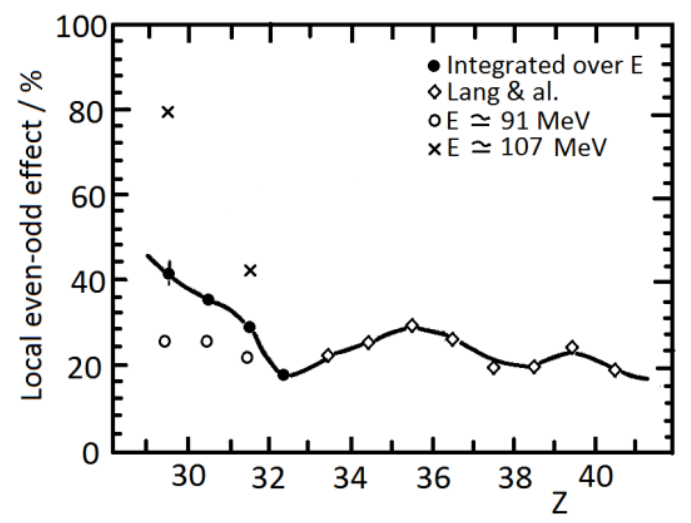

Fig. 5: Thermal neutron induced fission of ${ }^{235} U$. In the region of super-asymmetrical fission, the even-odd local effect on yields increases with the asymmetry of charge fragmentation. Taken from Ref. [13].

\section{Coulomb and even-odd effects in symmetric fragmentations}

Based on the Coulomb effect hypothesis, a prediction may be proposed. For symmetrical fragmentation, if one assumes scission configurations with spherical fragments, a higher separation of $C$ from $Q$ also occurs. Therefore, similarly to what happens with the asymmetric region, a greater even-odd effect may be expected. Indeed, there is a notorious even-odd effect in the curve of maximum kinetic energy for fragments in the mass region 104-108. See Fig. 3.

\section{Conclusion}

In cold fragmentation, the available energy is spent into Coulomb interaction energy and deformation energy, respectively. The fact that the even-odd effect in the maximum kinetic energy as a function of the mass or charge is lower than the even-odd effects in the available energy value suggests that, for the same deformation, even-even fragments need more energy than odd mass fragments.

Experimental results about cold fission suggest that, at scission, the total deformation energy of fragments competes with total intrinsic excitation energy: fragmentations which need high total deformation energy to occur will have low total intrinsic energy.

For the super-asymmetrical fragmentation the Coulomb interaction energy is much higher than the available energy. Those super-asymmetrical charge fragmentations need a super deformation to fulfill the energy balance condition; then a lower intrinsic excitation energy, which implies a lower probability for breaking of pairs of nucleons and, consequently, a higher even-odd effect of charge distribution is expected.

In the symmetric fission, the Coulomb interaction energy between supposedly spherical fragments is much higher than the $Q$-value. Then, similarly to the case of super-symmetric fragmentations, in symmetric region fragments should be quite deformed and, consequently, relatively high values of even-odd effects may be expected.

\section{References}

[1] W. Lang, H.-G. Clerc, H. Wohlfarth, H. Schrader y K.-H. Schmidt: Nucl. Phys. A345 (1980) 34-71

[2] C. Signarbieux, M. Montoya, M. Ribrag, C. Mazur, C. Guet, P. Perrin, y M. Maurel: J. Physique Lettres, 42 (1981) L-437 (1981)

[3] M. Montoya: Thesis, Doctorat d'Etat, Universidad París XI, Orsay (1981)

[4] M. Montoya, J. Physique 44 (1983) 785-790

[5] H. Nifenecker, G. Mariolopoulos et J.P. Bocquet, J. Physique Lett. 42 (1981) 527-529

[6] F. Gönnenwein and F. Börig: Nucl. Phys. A530 (1991) 27-57 
Revista ECIPerú

[7] M. Montoya: Z. Phys. A - Atoms and Nuclei, 319 (1984) 219-225.

[8] G. Simon, J. Trochon and C: Signarbieux; Fission Meeting; Arcachon (France); 14 - 17 Oct 1986; CEA-CONF 8860

[9] G. Audi and A.H. Wapstra: Nucl. Phys. A565 (1993) 1-65

[10] F. Gönnenwein: Physics Procedia, 47 (2013) 107-114

[11] M. Montoya, R.W. Hasse and P. Koczon: Z. Phys. A - Atoms and Nuclei 325 (1986) 357-362
Volumen 12 , número 1

Julio 2015

[12] M. Montoya: Revista Mexicana de Física, 60 (2014) 350-356

[13] J.L. Sida, P. Armbruster, M. Bernas, J.P. Bocquet, R.Brissot and H.R. Faust, Nucl. Phys, A502 (1989) 233c

E-mail:mmontoya@uni.edu.pe 